\title{
Statistical tuning of walfisch-bertoni pathloss prediction model based on building and street geometry sensitivity parameters in built-up terrains
}

\author{
Isabona Joseph ${ }^{1}$, Babalola Michael ${ }^{2}$ \\ ${ }^{1}$ Department of Basic Sciences, Benson Idahosa University, PMB.1100, Benin City, Nigeria \\ ${ }^{2}$ Department of Physics, Faculty of Physical Sciences, University of Benin, PMB.1154, Benin City, Nigeria
}

\section{Email address:}

josabona@yahoo.com,jisabona@biu.edu.ng

\section{To cite this article:}

Isabona Joseph, Babalola Michael. Statistical Tuning Of Walfisch-Bertoni Pathloss Prediction Model Based On Building And Street Geometry Sensitivity Parameters In Built-Up Terrains. American Journal of Physics and Applications. Vol. 1, No. 1, 2013 , pp. $10-17$. doi: 10.11648/j.ajpa.20130101.13

\begin{abstract}
One of the underlying difficulties with the application of a prediction pathloss model for any environment is that no two areas are identical in the composition of the buildings and terrain. A pathloss model developed by WalfischBertoni considers the impact of rooftops and building heights by using diffraction to predict average signal strength loss at street level. However, the error between the average path loss predicted by the model and that observed in practice will be smallest when the propagation environment conforms closely with the models assumptions (i.e. those urban environments exhibiting minimal variation in the height and separation of buildings). In particular, any building height variations can be expected to cause a significant error in the model predictions. Firstly, in this paper, we demonstrate the sensitivity impact of varying certain parameters on the performance $\mathrm{W} / \mathrm{B}$ model in the study environments. The goal is to give the user an idea of the value associated with altering parameters that are in fact adjustable and showing the significance of the unchangeable parameters in the loss calculations. Secondly, a new statistical tuning method is proposed by extending the technique of Walfisch- Bertoni model which is now valid for generalized conditions in the CDMA2000 signal propagation environments. The results showed that tuned pathloss data agree strongly with measured data in the different study locations.
\end{abstract}

Keywords: CDMA2000, Statistical Tuning Of Pathloss Model, Sensitivity of Impact Streets and Building Geometry

\section{Introduction}

The mobile radio channel, the air interface between the transmit antenna and receiving antenna introduces fundamental limitations on the performance of wireless communication systems. Unlike wireless channels, radio channels are extremely random and affected by various obstacles and movement of the receiver.

Most cellular radio systems operate in urban areas where there is no direct line-of-sight path between the transmitter and the receiver, and where the presence of high-rise buildings causes severe diffraction loss. Also, due to multiple reflections from various objects, the waves travel along different paths of varying lengths. The interaction between these waves causes multipath fading at a specific location, and the strengths of the waves decreases as the distance between the transmitter and receiver increases.
The model developed for one urban area may not give acceptable results for a different urban area since; each model has different parameters according to the area considered. Therefore, in order to study the nature of radiation pattern and quality of signal persisting in a particular region we definitely require certain kind of modeling to bring out the natural characteristics of the environment in to machine coordinated implementation and for the same we deploy radio propagation models.

Bertoni-Walfisch (W/B) model (also known as diffraction model) has not only considered the free space path loss, but also considered the diffraction loss along the path propagation, as well as the impact of roof and building height [1]. W/B model is used as ITU-R in the standard of IMT-2000. In urban environment, the frequency range is $800 \mathrm{MHz} \sim 2000 \mathrm{MHz}$ [2]. Besides the basic parameters such as the link frequency (f), the distance between transmitter and receiver $(\mathrm{R})$, the heights of transmitter and 
receiver antennas $\left(h_{T}\right.$, and $\left.h_{m}\right)$, there are two other key parameters: the height building $\left(\mathrm{H}_{\mathrm{b}}\right)$ and the distance between buildings $\left(d_{b}\right)$. The performance of $W / I$ model is greater than others because it uses both experimental and theoretical approaches. However, it is not appropriate to apply the model for every urban area, because $\mathrm{H}_{\mathrm{b}}$ and $\mathrm{d}_{b}$ parameters may also be different. Therefore, the specific circumstances of these two parameters must be considered when applies this model, while researches before have only considered a parameter situation [3], [4], [5].

\section{Statement of Problem}

The W/B model is first model which takes into consideration the effect of buildings on radio propagation channel in path-loss modeling [18]. The model assumes that propagation takes place over rows of buildings having equal heights and equal spacing arranged in a perfect grid (see section $\mathrm{C}$ for details). The model has also been adopted by International Telecommunication Union (ITU) for IMT2000/3G standard ([10], [11]. However, the error between the average path loss predicted by the model and that observed in practice will be smallest when the propagation environment conforms closely with the models assumptions (i.e. those urban environments exhibiting minimal variation in the height and separation of buildings). In particular, any building height variations can be expected to cause a significant error in the model predictions and this has been confirmed by the theoretical investigation study carry out in [7], [8], and [10].

In literature, some attempts have been made to improve the model's prediction accuracy in different propagation environments. In [16], effects of differences in height and buildings structures to the signal spread are studied to improve on the Walfisch and Bertoni model. Piazzi and Bertoni [9] find the spread loss model by assuming that the buildings which have same height and distance are located on uneven land. Chung and Bertoni [7] have presented a theoretical model. This model is improved by using the approach of Walfisch - Bertoni. The benefit of transmitter antenna height is also included in the model.

A tuning of "Bertoni-Walfisch" path loss model for GSM-900 MHz Network implemented in Turkey has been presented in [13]. The model is based on measurements for several areas in Istanbul. The path loss exponents $n$ for these areas were found in the range from 2 to 4.4. In [20], site-specific measurement based predictions method has been presented to adjust the W/B model for ThirdGeneration (3G) Systems radio channel in high-core building dominated urban environment. A Fuzzy adaptive neural network approach to tune path loss prediction accuracy of W/B in urban areas at GSM-900 band is contained in [14].

In this study, a precise optimisation method is proposed for the tuning the parameters of $\mathrm{W} / \mathrm{B}$ model to improve its accuracy in the UHF CDMA2000 propagation predictions in South-South, Nigeria.

\section{Research Methodology}

The objective of propagation model tuning is to obtain values for model parameters and land usage clutter such that they are in agreement with measured data.

Firstly, in this paper, the physical approximation deployed in W/B model is presented.

Secondly, the sensitivity of two key parameters of the W/B model such as the building height, $h_{B}$ and distance between building, $d_{B}$ are studied via first order statistics, to show how both parameters impact the performance of the model in different propagation terrain. This is followed by coming up with a least square tuning algorithm to adjust the model parameters in such a way that the difference between measured pathloss data from the actual propagation environment and the respective model predictions is minimized. The algorithm which performs an iterative differential correction on the W/B model to produce what should be a better representation of the real building geometry of the propagation environment is implemented using Java Script program.

\section{Physical Approximation Deployed in Walficsh-Bertoni Model}

The model considers the buildings in the vertical plane between the transmitter and the receiver, which are characterized as diffracting half-screens of equal heights, widths and separations. At the terminal, the received field is composed of two rays - the direct multiple diffracted and diffracted-single-reflected one - which are vector summed to account for their phases. Walfisch and Bertoni studied diffraction over multiple edges using Kirchhoff - Huygens theory integrating for each half screen.

For calculating the loss along buildings the model starts by using the Repeated Kirchhoff Integral for uniform parallel screens which is an application of scalar diffraction theory with some approximations [18]. In the case when there are uniform screens with uniform heights, the field at the plane of screen can be written in terms of the incident wave coming towards the first screen. This can be done by expressing the field at each consecutive screen in terms of the field at the previous one as shown in figure 1

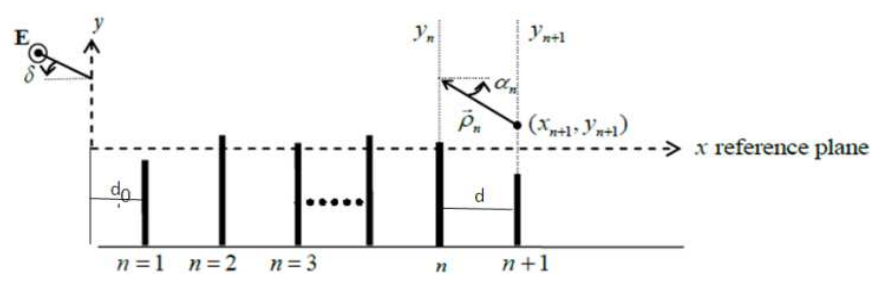

Figure 1. Parallel Screens with varying Height

Going from the first screen $n$ to screen $n+1$, the field can 
be written as [19],

$$
E\left(d, y_{1}\right)=\frac{e^{j \frac{\pi}{4}} e^{-j k d}}{\sqrt{\lambda d}} \int_{0}^{\infty} E\left(d_{o}, y\right) e^{\frac{-j k\left(y_{1}-y\right)^{2}}{2 d}} d y
$$

And for the second screen one has

$$
E\left(2 d, y_{2}\right)=\frac{e^{j \frac{\pi}{4}} e^{-j k d}}{\sqrt{\lambda d}} \int_{0}^{\infty} E\left(d, y_{1}\right) e^{\frac{-j k\left(y_{2}-y_{1}\right)^{2}}{2 d}} d y_{1}
$$

And for the third screen one has

$$
E\left(3 d, y_{3}\right)=\frac{e^{j \frac{\pi}{4}} e^{-j k d}}{\sqrt{\lambda d}} \int_{0}^{\infty} E\left(2 d, y_{2}\right) e^{\frac{-j k\left(y_{2}-y_{1}\right)^{2}}{2 d}} d y_{2}
$$

And so forth and so on until the last screen $y_{N+1}$

$$
E\left((N+1) d, y_{N+1}\right)=\frac{e^{j N \frac{\pi}{4}} e^{-j k N d}}{(\lambda d)^{N / 2}} \int_{0}^{\infty} d y_{1} \int_{0}^{\infty} d y_{2} \int_{0}^{\infty} d y_{3} \ldots \int_{0}^{\infty} d y_{N} E\left(d_{1}, y_{1}\right) e^{-j \frac{k}{2 d} \sum_{n=1}^{n}\left(y_{n+1}-y_{n}\right)^{2}}
$$

With a parameter,

$$
V_{n}=y_{n} \sqrt{\frac{j k}{2 d}}
$$

and the identity,

$$
j=e^{j \frac{\pi}{2}}
$$

$$
E\left((N+d) d, y_{N+1}\right)=\frac{e^{-j k N d}}{\pi^{N / 2}} \int_{0}^{\infty} d v_{1} \int_{0}^{\infty} d v_{2} \int_{0}^{\infty} d v_{3} \ldots \int_{0}^{\infty} d v_{N} E\left(d, y_{1}\right) e^{-\sum_{n=1}^{n}\left(v_{n+1}-v_{n}\right)^{2}}
$$

The incident plane wave in 2D coordinate can be written as,

$$
E\left(d, y_{1}\right)=E_{0} e^{-j k . r}=E_{0} e^{-j k(d \cos \delta y \sin \delta)}
$$

For small angles and unit amplitude $E\left(d, y_{1}\right) \approx e^{-j k d} e^{j k y_{1} \sin \delta}$ and by Taylor series expansion

$$
E\left(d, y_{1}\right) \approx e^{-j k d} \sum_{m=0}^{\infty} \frac{1}{m !}\left(j k y_{1} \sin \delta\right)^{m}(10)
$$

Introducing a dimensionless parameter,

$$
g=\sin \delta \sqrt{\frac{d}{\lambda}} \text { where } V_{1}=y_{1} \sqrt{\frac{j k}{2 d}}
$$

Yields,

$$
E\left(d, y_{1}\right)=e^{-j k d} \sum_{m=0}^{\infty} \frac{1}{m !}\left(2 g \sqrt{j \pi} V_{1}\right)^{m}
$$

At the screen $\left(x_{N+1}, y_{N+1}\right)$ for the point where $y_{N+1}=0$ and $x_{N+1}=(\mathrm{N}+1) \mathrm{d}$, the expression for $\mathrm{E}\left(x_{N+1}, 0\right)$ becomes.

$$
\begin{gathered}
E\left(d, y_{1}\right)=e^{-j k d} \sum_{m=0}^{\infty} \frac{1}{m !}\left(2 g \sqrt{j \pi} V_{1}\right)^{m}, \\
E((N+1) d, 0)=\frac{e^{-j k(N+1) d}}{\pi^{N / 2}} \int_{0}^{\infty} d v_{1} \int_{0}^{\infty} d v_{2} \int_{0}^{\infty} d v_{3} . \\
. . \int_{0}^{\infty} d v_{N} \sum_{m=0}^{\infty} \frac{1}{m !}\left(2 g \sqrt{j \pi v_{1}}\right)^{m} e\left(-v_{1}^{2}+2 \sum_{N=1}^{N-1}\left(v_{n+1} v_{n}\right)-2 \sum_{n=2}^{N} v_{n}^{2}\right)
\end{gathered}
$$

This result can be written in terms of the Boersma function which is in general defined as [19]

$$
\begin{aligned}
& I_{N, m}(\beta)=\frac{1}{\pi^{N / 2}} \int_{0}^{\infty} d v_{1} \int_{0}^{\infty} d v_{2} \int_{0}^{\infty} d v_{3} \\
& \ldots \int_{0}^{\infty} d v_{N}\left[v_{1}^{m} e^{\left(-\beta v_{1}^{2}+2 \sum_{n=1}^{N-1}\left(v_{n+1} v_{n}\right)^{-2} \sum_{n=2}^{N} v_{n}^{2}\right)}\right],
\end{aligned}
$$


Thus for $\beta=1$

Boersma evaluates $I_{N, m}(\beta)$ using a recursion relation for $m$ $\geq 2$ as follows:

$$
\begin{aligned}
& E((N+1) d, 0)=e^{-j k(N+1) d} \sum_{m=0}^{\infty} \frac{1}{m !}(2 g \sqrt{j \pi})^{m} I_{N, m}(1),(16) \\
& I_{N, m}(\beta)=\frac{N(m-1)}{2(N+1)^{\beta-1}} I_{N, m-2}(\beta)+\frac{1}{2 \sqrt{\pi}(N+1)^{\beta-1}} \sum_{n=\beta-1}^{N-1} \frac{I_{n, m-1}(\beta)}{\sqrt{N-n}}
\end{aligned}
$$

For $\beta=1$ the initial value is given as:

$$
I_{0, m}(1)=\left\{\begin{array}{lll}
1 & \text { for } & m=0 \\
0 & \text { for } & m>0
\end{array}\right\} ; I_{N, 0}(1)=\frac{(1 / 2)_{N}}{N !} ; I_{N, 1}(1)=\frac{1}{2 \sqrt{\pi}} \sum_{n=0}^{N-1} \frac{(1 / 2) n}{n ! \sqrt{N-n}},
$$

Where $(1 / 2)_{\mathrm{n}}$ is the Pochhammer symbol which is defined as,

$$
(p)_{0}=1 ;(p)_{1}=p ;(p)_{n}=p(p+1) \ldots(p+n-1)
$$

For propagation parallel to the rooftops, with the angle of incidence $\delta=0^{\circ}$, the parameter $g$ vanishes due to equation (12) and,

$$
E((N+1) d, 0)=e^{-j k(N+1) d} I_{N, 0}(1)
$$

Using the asymptotic approximation for $I_{N, 0}(1)=\frac{(1 / 2) N}{N !}$ one can finally obtain the following result:

$$
E\left(x_{N+1}, 0\right)=e^{-j k(N+1) d} \frac{(1 / 2)_{N}}{N !} \approx e^{-j k(N+1) d} \frac{1}{\sqrt{\pi N+1}} .
$$

This expression implies that the amplitude of the field decrease monotonically as $1 / \sqrt{N}$

As the plane wave is diffracted past a series of absorbing screens for low glancing angle, the field incident on successive edges is found to decrease initially to a value $\mathrm{g}$, called the settled field. This settling process is associated with the end effects of the starting screens. Walfisch and Bertoni, [18], using polynomial fitting curve showed that the settled value $g$, gives the reduction of the rooftops fields resulting from diffraction of previous rows of buildings by:

$$
P(g) \approx 0.1\left(\frac{g}{0.03}\right)^{0.9} \text { for } 0.015<g<0.4
$$

Considering the expression $g=\sin \delta \sqrt{\frac{d}{\lambda}}$ in (12) for (22), we have,

$$
P(g)=0.1\left(\frac{\sin \delta \sqrt{\frac{d}{\lambda}}}{0.03}\right)^{0.9}
$$

The pathloss along the rooftops can then written in terms of the field reduction as,

$$
P L_{\text {rooftops }}=P(g)^{2}=\left[0.1\left(\frac{\sin \delta \sqrt{\frac{d}{\lambda}}}{0.03}\right)^{0.9}\right]^{2}
$$

Here, $\sin \delta$ can be written in terms of the BS transmitter height $\mathrm{h}_{\mathrm{T}}$, the building height, $\mathrm{h}_{\mathrm{B}}$, and the distance $\mathrm{R}$ as,

$$
\sin \delta=\frac{h_{T}-H_{g}}{R}
$$

Therefore expression in equation (23) becomes,

$$
P L_{\text {rooftops }}=0.01\left(\frac{h_{T}-H_{B}}{0.03 R}\right)^{1.8}\left(\frac{d}{\lambda}\right)^{0.9}
$$

To find the reduction of the field power diffracted from the last screen (building) down to the street level, Ikegami's approach can be used. It states that the spatial average power over a distance is approximately the sum of the individual ray powers. Another fact is that the plane waves incident on the building edges act as a source of cylindrical waves for the mobile level.

One can then come up with an estimate by using that the power density of cylindrical waves is inversely proportional to the path distance $\rho_{i}$ from the building edge to the mobile, and proportional to the square of the diffraction coefficient for an absorbing wedge $\left|D\left(\theta_{i}\right)\right|^{2}$. The diffraction coefficients differ with boundary conditions but for small angles they approach the same dependence. For simplicity, using the Fresnel coefficients, 


$$
\left|D\left(\theta_{i}\right)\right|^{2}=\frac{1}{2 \pi k}\left(\frac{1}{\left|\theta_{i}\right|}-\frac{1}{2 \pi-\left|\theta_{i}\right|}\right)^{2},
$$

The diffraction from the rooftop down the street level can be written as follows:

$$
\begin{aligned}
& P L_{\text {down }}=\frac{1}{2 \pi k \rho_{2}}\left(\frac{1}{\left|\theta_{1}\right|}-\frac{1}{2 \pi-\left|\theta_{1}\right|}\right)^{2} \\
& +\frac{|\Gamma|^{2}}{2 \pi k \rho_{1}}\left(\frac{1}{\left|\theta_{2}\right|}-\frac{1}{2 \pi-\left|\theta_{2}\right|}\right)^{2}
\end{aligned}
$$

Considering the Figure 2, with the assumptions $\rho_{2}=3 \rho_{1}$ and $|\Gamma|^{2}=0.1$, then the rays, the rays have nearly equal amplitudes [19]. Thus,

$$
\begin{aligned}
& P L_{\text {down }}=\frac{1}{\pi k \rho_{1}}\left(\frac{1}{\left|\theta_{1}\right|}-\frac{1}{2 \pi-\left|\theta_{1}\right|}\right)^{2} \\
& \approx \frac{1}{\pi k \rho_{1} \theta_{1}^{2}} \approx \frac{\lambda \rho_{1}}{2 \pi^{2}\left(H_{b}-h_{m}\right)} .
\end{aligned}
$$

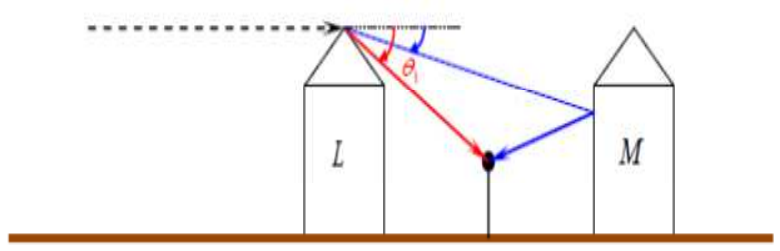

Figure 2. Geometry for finding the loss down at the street level

Finally, the total pathloss can be written as,

$$
\begin{aligned}
& P L_{\text {total }}=\left(\frac{\lambda}{4 \pi R}\right)^{2} P(g)^{2} \frac{\lambda \rho_{1}}{2 \pi^{2}\left(H_{b}-h_{m}\right)} \\
& =\frac{5.51}{32 \pi^{4}} \frac{\left(h_{r}-H_{g}\right)^{1.8} \rho_{1} d^{0.9}}{\left(H_{B}-h_{m}\right)^{2}} \frac{\lambda^{2.1}}{E^{3.8}}
\end{aligned}
$$

Equation (29) can be expressed in decibels as follows:

$$
\begin{aligned}
& P L_{\text {total }}=89.5-10 \log \left[\frac{\rho_{11} d^{0.9}}{\left(H_{B}-h_{m}\right)^{2}}\right] \\
& +21 \log f_{m}-18 \log \left(h_{T}-H_{B}\right)+38 \log R_{k}
\end{aligned}
$$

where

$$
\rho_{1}=\sqrt{\left(\frac{d}{2}\right)^{2}+\left(H_{B}-h_{m}\right)^{2}}
$$

$H_{b}$ : Building height in meters.

$h_{m}$ : Mobile height in meters.

\section{Sensitivity Analysis}

Sensitivity analysis is a method to estimate the changes brought into the system through small changes in the parameters of the model [15]. In this way one may estimate both the robustness of the model against small changes, as well as identify possibilities of bringing a certain desired changed in the system

Here, firstly, the pathloss sensitivity influenced by parameters $h_{B}$ and $d_{B}$ are examined via simulation. Figure 3 shows path loss versus the height of building for the different the distance of base station transmitter and mobile receiver. Fig. 4 also shows the relation of path loss and the parameter of building spacing $\left(d_{B}\right)$. Also, the path loss influenced by joint parameters $h_{B}$ and $d_{B}$ is shown in Fig. 5 . It can be seen clearly from Fig. 3 to Fig. 5, that these parameters are very important in the calculation of path loss for mobile communication in built-up terrains.

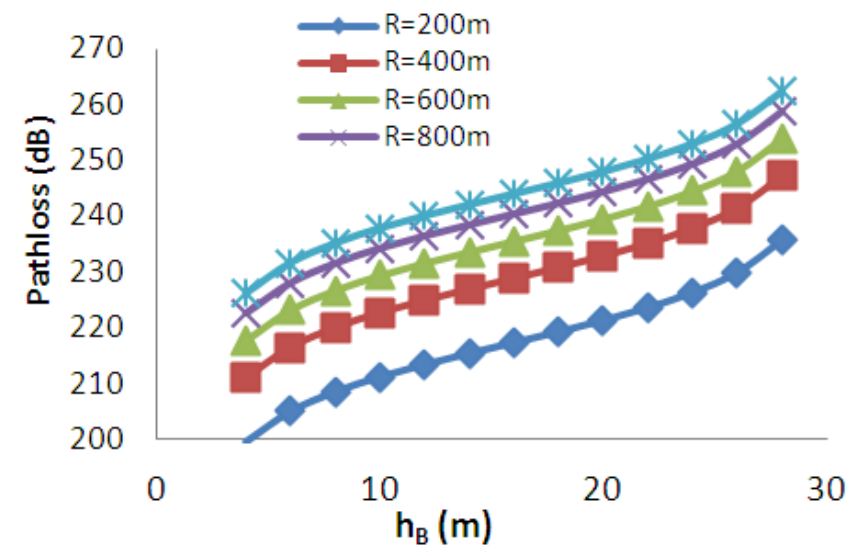

Figure 3. The sensitivity of pathloss as a function of $h_{B}$ for $h_{m}=1.5 \mathrm{~m}$, $d_{B}=20 \mathrm{~m}, h_{T}=30 \mathrm{~m}$, and $f=900 \mathrm{M}$

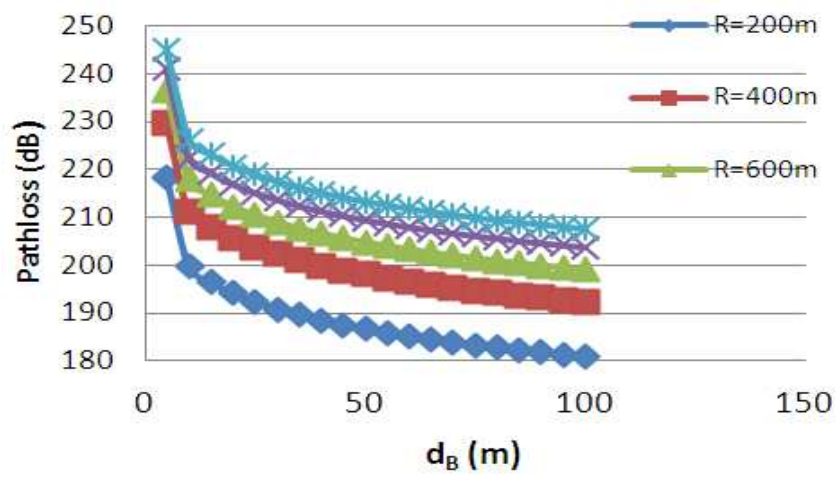

Figure 4. The sensitivity of pathloss as a function of $d_{B}$ for $h_{m}=1.5 \mathrm{~m}$, $h_{B}=10 \mathrm{~m}, h_{T}=30 \mathrm{~m}$, and $f=900 \mathrm{MHz}$ and

$f_{\mathrm{m}}$ :

$h_{T}$ :
Frequency in $\mathrm{MHz}$.

Antenna Height in meters. 


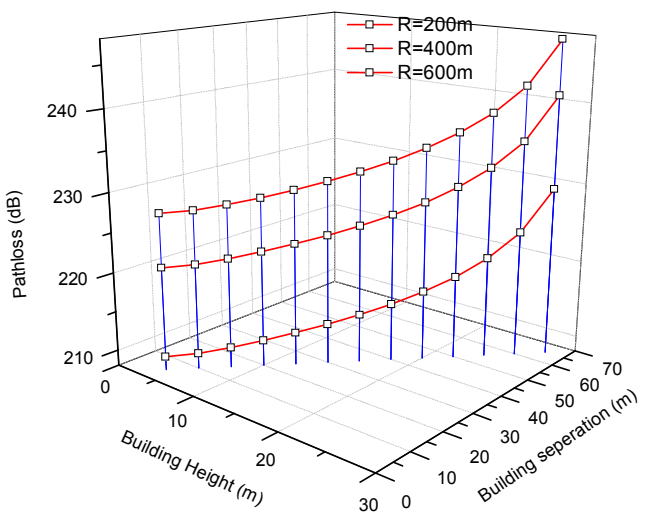

Figure 5. The sensitivity of pathloss as a function of varied $h_{B}=10 \mathrm{~m}$ and $d_{B}$, for $h_{m}=1.5 \mathrm{~m}, h_{T}=30 \mathrm{~m}$, and $f=900 \mathrm{MHz}$.

\section{Model Parameter Tuning Approach}

In order to determine the building height $\left(h_{B}\right.$ and the distance between building $\left(d_{B}\right)$ parameters statistically, measurements campaign was conducted on a $3 \mathrm{G}$-based cellular networks, transmitting over the CDMA2000 air interface, in the $1900 \mathrm{MHz}$ band. The field measurement was done at different period of the year at Benin, Uyo and Port Harcourt city and 9 BS cell sites selected in the three locations of study.

To achieve of the study, an automatic parameter tuning algorithm was then employed to tune the $h_{B}$ and $\left(d_{B}\right)$ parameters in such a way that the difference between measured pathloss data from the actual propagation environment and the respective model predictions is minimized in a least squares sense. The algorithm is implemented using Java Script program (see the appendix section for details). This approach enabled us to determine the correct $\left(h_{B)}\right.$ and $\left(d_{B}\right)$

\section{Results and Discussion}

Using the statistical tuned results, W/B formula can be simplified and generalized each for the three built-up urban environments (Benin, Port Harcourt and Uyo) by the expressions in equations (32) - (34).

$$
\begin{gathered}
P L_{\text {total }}=89.5+21 \log f_{m}-18 \log \left(h_{T}-6\right)+38 \log R_{k} \\
-10 \log \left[\frac{4.26\left(\sqrt{(2.25)^{2}+\left(6-h_{m}\right)^{2}}\right)}{\left(6-h_{m}\right)^{2}}\right] \\
P L_{\text {total }}=89.5+21 \log f_{m}-18 \log \left(h_{T}-5\right)+38 \log R_{k} \\
-10 \log \left[\frac{7.23\left(\sqrt{(4.5)^{2}+\left(5-h_{m}\right)^{2}}\right)}{\left(5-h_{m}\right)^{2}}\right]
\end{gathered}
$$

$$
\begin{gathered}
P L_{\text {total }}=89.5+21 \log f_{m}-18 \log \left(h_{T}-7\right)+38 \log R_{k} \\
-10 \log \left[\frac{5.02\left(\sqrt{(6)^{2}+\left(7-h_{m}\right)^{2}}\right)}{\left(7-h_{m}\right)^{2}}\right]
\end{gathered}
$$

Similarly, the graphs in Fig. 6 to Fig. 8 are plotted to examine how well the results of the tuned pathloss model data go with that of measured pathloss data.

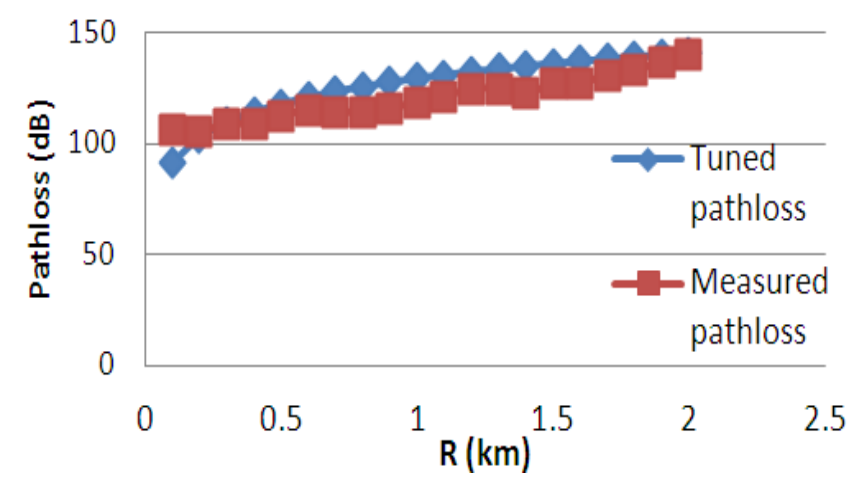

Figure 6. Measured versus tuned pathloss as a function distance in Benin

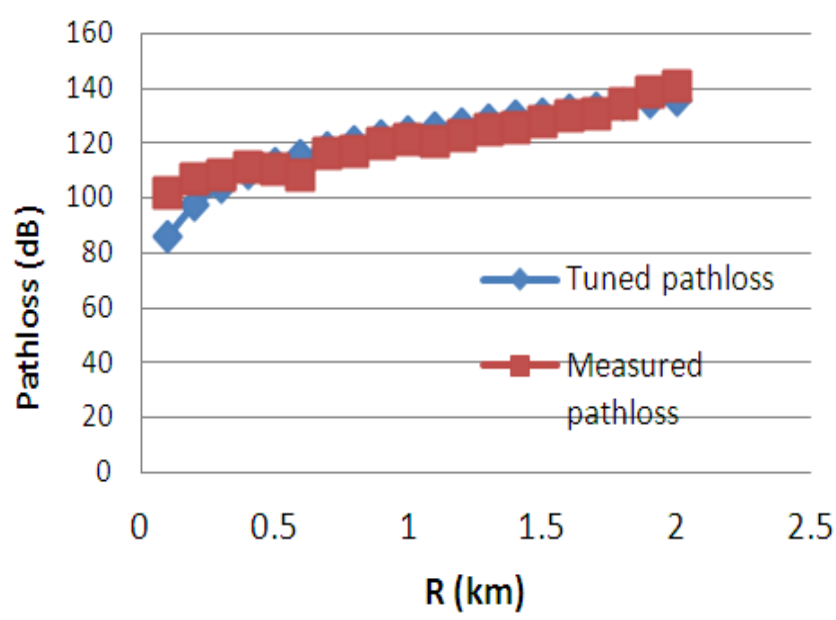

Figure 7. Measured versus tuned pathloss as a function distance in Uyo

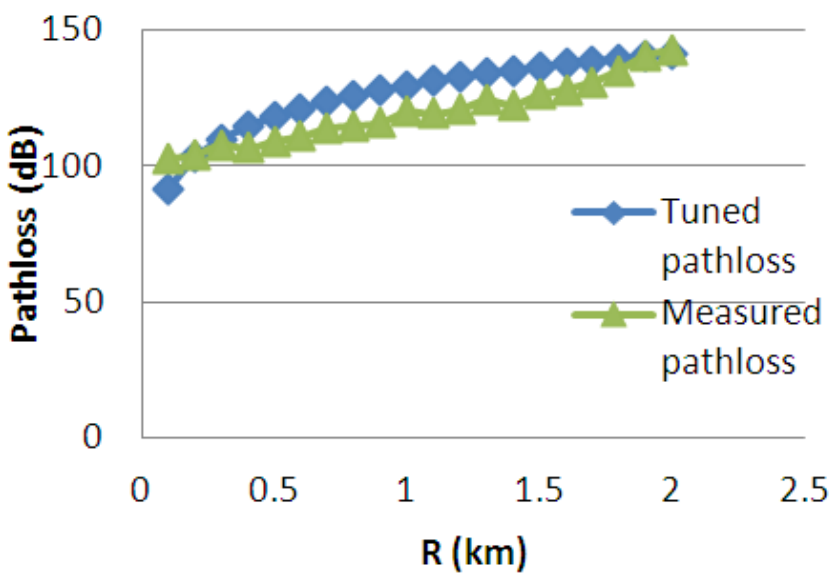

Figure 8. Measured versus tuned pathloss as a function distance in Port Harcourt 
It can be clearly observed from the above graphs that tuned pathloss data agree strongly with measured data. Thus, the tuned W/B models can be used for signal pathloss propagation prediction in the different studied environments.

\section{Conclusion}

The lack of a single compact model that can be used to predict signal pathloss for different urban areas is one of the most important problems. Walfisch- Bertoni model is a semi deterministic model for estimating the pathloss in built-up urban environment over the frequency range of 800-2000 MHz. There are two key parameters that are used in this model to calculate path loss. The parameters are the height and the distance between the buildings. In this paper, a new statistical tuning method is proposed by extending the technique of W/B model which is now valid for generalized conditions in the CDMA2000 signal propagation environments. The results showed that tuned pathloss data agree strongly with measured data in the different study location.

\section{Appendix}

The Java Script program performs an iterative differential correction on the W/B model to produce what should be a better representation of the real propagation environment.

Being $y_{i}$ a collection of measurements and $L_{i}$, the collection of predictions, the sum of the squares of the residuals is:

$$
S=\sum_{i=1}^{n}\left(y_{i}-L\right)^{2}=0
$$

According to the method, when the sum is minimum the model adjusts to the measurements.

The predictions, $L_{i}$ depend on some variables $p_{1}, p_{2}$, (Eq. A2), which suffers some changes to obtain the best approximation. The process to solve the problem is, first, to calculate the derivates of $\mathrm{S}$ with respect each variable of $L_{i}$ and make the resulting expressions equal to zero (Eq. A2). After this conversion results an equation system whose solution is the group of values of the variables to achieve the best approximation

$$
L_{i}=89.5+21 \log f_{m}-18 \log \left(h_{T}-P_{1}\right)+38 \log R_{k}-10 \log \left[\frac{\left(P_{2}\right)^{0.9}\left(\sqrt{\left(P_{2}\right)^{2}+\left(P_{1}-h_{m}\right)^{2}}\right)}{\left(P_{1}-h_{m}\right)^{2}}\right]
$$

The process is iterative and the core iterative process using the algorithm summarized as follows:

(a) Enter the number of data points: 20

(b) Enter the number of independent variables: $\sqrt{1}(0-$ 8 ; usually 1)

(c) Enter the number of parameters: $\sqrt{2}(1-8)$

Enter the Prediction model for the function to be fitted: $Y=\longdiv { \text { A+B*LOG10(f)-C*LOG10(k)+D*LOG10(x)-E*LOG10P) } }$

Perform appropriate filtering on the measurement data; to towards that end, we conduct measurements by taking a number of readings at a particular location and obtained the average. This approach is referred to as filtering (i.e. filtering the variations)

Enter the $[x, y]$ filtered measurement data:

\begin{tabular}{|l|l|}
\hline $0.1,101.33$ & \\
$0.2,104.67$ & \\
$0.3,106.67$ & \\
$0.4,106.67$ & \\
$0.5,106.33$ & \\
$0.6,107.67$ & \\
$0.7,112.67$ & \\
$0.8,114.60$ & \\
0 & \\
\hline
\end{tabular}

Perform a single iteration cycle, and observe how the parameters change in the boxes above. Also look at the RMS Error and the Output area below.
RMS Err $=4.22$

Is Error satisfactorily acceptable

If the new parameter values seem reasonable, or perform iteration again, and continue until the parameters converge.

\section{References}

[1] Cavdrih I. H, "A Statistical Approach to Bertoni-Walfisch Propagation Model for Mobile Radio Design in Urban Areas". IEEE VTC 52 nd, Vol.1, pp. 279-283, 2001.

[2] "Coverage prediction for mobile radio systems operation in the $800 / 900 \mathrm{MHz}$ Frequency Range". Special issue of IEEE Transactions on Vehicular Technology, 37(1):66-68, 1988. [Online] Available at: http://ieeexplore.ieee.org

[3] Dongya S, Yihuai Y, and Taijun L, "Physical-statistical Propagation Model on the Land Mobile Communications", Proceedings of 2005 Asia-Pacific Microwave Conference, vol.4, pp.2349-22352, Dec. 2005, Suzhou, China, IEEE Press.

[4] Yang Y, and Dongya. S and Taijun L,'Physical-statistical Propagation Model on the Land Mobile Communications",Journal of Yunnan University, 2006, 9.

[5] Gong K.,and Xu R. "Modeling of 3D In-Building Propagation Effects by Ray Tracing Technique". IEEE AP-S, 6, 1995 .

[6] C. Chrysanthou. and H. L. Bertoni, "Variability of Sector 
averaged Signals for UHF Propagation in Cities", IEEE Transaction Vehicular Technology, vol. 39. No. 4, pp. 352358 , November, 1990

[7] H. Chung and H. L. Bertoni, "Range-dependent path-loss model in residential areas for the VHF and UHF bands", IEEE Transactions, Antennas and propagation, vol. 50, pp. 1-11, January, 1993.

[8] S. Saunders, and F. Bonar, "Mobile radio propagation in built-up areas: A numerical model of slow fading," in IEEE Vehicular Technology Symposium, pp. 295-300, May, 1991.

[9] L. Piazzi. and H.L Bertoni, "Effect of Terrain on Pathloss in Urban Environments for Wireless Applications", IEEE Transaction on Antennas and Propagation, Vol. 46, No. 8, pp.1138-1147, August, 1998.

[10] L. Meiling, L. Nikolai, V. Guillaume, and Guillaume D. 1. R "On Predicting Large Scale Fading Characteristics with the MR-FDPF Method, 6th European Conference on Antennas and Propagation (EECAP) Prague: Czech Republic, March, 2012.

[11] S. Kolyaie, M. Yaghooti, and G. Majidi, "Analysis and Simulation of Wireless Signal Propagation Applying Egotistical Techniques, Archives of Photogrammetry", Cartography and Remote Sensing, Vol. 22, pp. 261-270, 2011.

[12] F.D. Alotaibi. and A.A, "TETRA Outdoor Large- Scale Received Signal Prediction Model in Riyadh City-Saudi Arabia", IEEE Wireless and Microwave Technology Conference (WAMICON), USA, pp. 4-5, Dec. 2006.
[13] T.E. Dalkilic, B.Y.S. Hanci, and A.S. Apatdin, "Fuzzy adaptive neural network approach to tune path loss prediction accuracy of W/B in Urban areas at GSM-900 band", Turkish Journal of Electrical Engineering and Computer Science, Vol.18, No.6, pp 1077-1094, 2010.

[14] Y.B Hanci, and I.H. Cavdar, "Mobile Radio Propagation Measurements and Tuning the Path Loss Model in Urban Areas at GSM-900 Band in Istanbul-Turkey", IEEE Vehicular Technology Conference (VTC2004), Vol.1, September, $139-143,2004$.

[15] Pathak, R and Katiyar, M “ Performance and Sensitivity of pathloss Models for WiMAX Signals" Conference on Advances in Communication and Control Systems 2013(CASC25S 2013)

[16] Recommendation M.1455 "Key characteristics for the international mobile telecommunications 2000 (IMT-2000) Radio Interface", 2000.

[17] Recommendation M.1225 "Guidelines for evaluation of radio transmission technologies for IMT-2000”, 2000.

[18] J. Walfisch and H.L. Bertoni. "Theoretical Model of UHF Propagation in Urban Environments", IEEE Transaction on Antenna and Propagation, vol. 36, pp.1788-1796, December, 1998.

[19] Bertoni H.L., Radio Propagation for Modern Wireless Systems, Prentice-Hall PTR, Upper Saddle River, NJ, 2000.

[20] S. A. Toricco, and H.L Bertoni, "Predicting the Radio Channel for Third-Generation Systems", White paper, COM search Bulletin TP-100328-EN (04/05), pp.1-12, 2005. 